Pacific Journal of Mathematics

A METRIC BASIS CHARACTERIZATION OF EUCLIDEAN 


\title{
A METRIC BASIS CHARACTERIZATION OF EUCLIDEAN SPACE
}

\author{
Grattan P. MURPhy
}

\begin{abstract}
In $E^{n}$ there is exactly one line containing a given pair of points and exactly one $k$-flat containing a given $k$-simplex $(k+1$ points not contained in a lower dimensional space). The purpose of this paper is to prove converses of these propositions in the setting of complete, convex metric spaces. The most striking of these is given in Theorem 1 where it is proved that a complete, convex metric space which can be uniquely determined by any pair of points must be isometric with a subset of the real line. Theorem 2 is a higher dimensional analogue of this theorem. Metric characterizations of $E^{1}$ and $E^{n}$ are derived from these results.
\end{abstract}

The distance between points $p$ and $q$ will be denoted by $p q$. A metric space $M$ is convex if for each pair of distinct points $p, q$ there exists a point $r$ with $p \neq r \neq q$ and $p q=p r+r q . \quad M$ is externally convex if there exists a point $s$ with $p \neq s \neq q$ and $s q=s p+p q$. For the remainder of this paper a space $M$ will always mean a complete and convex metric space.

Definition 1. A subset $B$ of $M$ is called a metric basis for $M$ if $x z=y z$ for every $z$ in $B$ implies $x=y$.

It is easy to show that each pair of distinct points in $E^{n}$ is a metric basis for the line it determines and that the vertices of a nondegenerate simplex form a metric basis for $E^{n}$.

The fact that each pair of points forms a metric basis for $E^{1}$ is almost characteristic of that space, as the following theorem shows.

THEOREM 1. If each pair of distinct points of $M$ forms a metric basis for $M$, then $M$ is isometric with a subset of $E^{1}$.

Proof. First we show that each three points of $M$ are collinear. Suppose $M$ contains points $p, q$, and $r$ which are not collinear. By a theorem of Menger [5], each two points of $M$ are the end points of a metric segment (isometric image of a line segment) in M. Let $p q \geqq p r$ and denote by $S(p, q)$ a fixed segment joining $p$ and $q$. By the continuity of the metric and the fact that $S(p, q)$ is connected, it follows that there is a point $x$ on $S(p, q)$ with $x q=x r$. If $m$ is a 
midpoint of $q$ and $r$, then $m q=m r$. There are two cases to consider, $x=m$ and $x \neq m$. If $x=m$, then suppose without loss of generality that $x p \geqq x r$. Using arguments similar to those above, we find a point $y$ between $x$ and $p$ with $x y=x r$. Since $q \neq x,\{q, x\}$ is a metric basis for $M$. But $q y=q r$ and $x y=x r$ imply $y=r$ from which it follows that $r$ is between $p$ and $q$, contradicting the nonlinearity of that triple. If $x \neq m$, then $\{x, m\}$ is a metric basis for $M$ and $x q=x r$ and $m q=m r$ imply $q=r$, a contradiction. This establishes that each three points of $M$ are collinear. If $M$ is a singleton, then the theorem follows immediately. Otherwise, $M$ contains at least five points and every three points of $M$ are isometric with three points of $E^{1}$. It is well known that such spaces are isometrically contained in $E^{1}[1, \mathrm{p} .117]$.

Since the metric space $M$ of Theorem 1 is complete and convex, its image in $E^{1}$ must be a singleton, a closed interval, a closed half-line, or $E^{1}$, itself. This suggests that, with very few additional assumptions we may characterize $E^{1}$. Indeed, we have:

COROLlARY 1.1. $E^{1}$ is characterized among all complete, convex, and externally convex spaces containing at least two points by the property that each two distinct points form a metric basis for the space.

Definition 2. $A$ subset $B$ of a metric space $M$ is a complete metric basis for $M$ if $B$ is a metric basis for $M$ and if every isometry of $B$ with another subset of $M$ may be extended to a motion of $M$ (an isometry of $M$ to $M$ ).

COROLlARY 1.2. $E^{1}$ is characterized among all complete and convex metric spaces containing at least two points by the property that each two distinct points form a complete metric basis for the space.

The aim of this part is to generalize Theorem 1 and its corollaries to $E^{n}$. We formalize the properties of bases in $E^{n}$ with the following definitions.

Definition 3. A metric space has the $n$-basis property if every $n+1$ points, not isometric with $n+1$ points of $E^{n-1}$, form a metric basis for $M$.

Definition 4. A metric space has the complete $n$-basis property if every $n+1$ points, not isometric with $n+1$ points of $E^{n-1}$, form a complete metric basis for $M$.

A "first approximation" to a generalization to Theorem 1 might be: If $M$ has the $n$-basis property, then $M$ is isometric with a subset of $E^{n}$. The hyperbolic plane, however, is a counterexample for $n=$ 
2. The axioms of a metric space imply that every triple of points are isometric with some three points in $E^{2}$. This is a subtle assumption in Theorem 1. In order to prove a generalization of Theorem 1 by methods similar to those of the proof of Theorem 1, we need to have certain $n+2$-tuples of points of $M$ congruently contained in $E^{n+1}$. One assumption which will insure such congruences for complete and convex metric spaces is the euclidean four point property.

DEFINITION 5. A metric space has the euclidean four point property if each four points of $M$ are isometric with four points of $E^{3}$.

THEOREM 2. If $M$ has the euclidean four point property and the $n$-basis property, then $M$ is isometric with a subset of $E^{n}$.

Proof. Wilson $[7,8]$ showed that, in $M$, the euclidean four point property implies that every $k+1$ points of $M$ are isometric with $k+1$ points of $E^{k}$. If every $n+2$ points of $M$ are isometric with $n+2$ points of $E^{n}$, then $M$ is isometric with a subset of $E^{n}$ since $E^{n}$ has quasicongruence order $n+2[1$, p. 117]. Likewise, if each $n+1$ points of $M$ are isometric with $n+1$ points of $E^{n-1}$ then $M$ is isometric with a subset of $E^{n-1}$. If not, then there are $n+2$ points $x_{0}, \cdots, x_{n+1}$ of $M$ which are isometric with $n+2$ points of $E^{n+1}$, but not with $n+2$ points of $E^{n}$. Let $y_{1}$ be the midpoint of $x_{0}$ and $x_{1}$, and define $y_{i}(2 \leqq i \leqq n+1)$ in the following way: if $x_{1} x_{i}=x_{0} x_{i}$, then $y_{i}=x_{i}$; if not and $x_{1} x_{i}>x_{0} x_{i}$ $\left(x_{1} x_{i}<x_{0} x_{i}\right)$, then $y_{i}$ is a point between $x_{i}$ and $x_{1}\left(x_{0}\right)$ with $x_{0} y_{i}=x_{1} y_{i}$. If $y_{1}, \cdots, y_{n+1}$ is not isometric with a set of $n+1$ points of $E_{n-1}$, then it is a metric basis for $M$ and $x_{0}=x_{1}$, which contradicts the assumption that $x_{0}, \cdots, x_{n+1}$ are not isometric with $n+2$ points of $E^{n}$. So assume $y_{1}, \cdots, y_{n+1}$ are isometric with $n+1$ points of $E_{n-1}$. Consider the set $S=Y \cup X=\left\{y_{i} \mid 1 \leqq i \leqq n+1\right\} \cup\left\{x_{j} \mid 0 \leqq j \leqq n+1\right\} . \quad Y \quad$ is isometric with a subset of $E^{n-1}$ so $Y \cup\left\{x_{0}\right\}$ is isometric with a subset of $E^{n}$. Since $x_{1}$ lies on the line joining $x_{0}$ and $y_{1}, Y \cup\left\{x_{0}, x_{1}\right\}$ is isometric with a subset of $E^{n}$. Likewise, for any $i>1, x_{i}$ is collinear with $y_{i}$ and $x_{0}$ or $x_{1}$ or else $x_{i}=y_{i}$. We then have $S$ isometric with a subset of $E^{n}$. But $X \subseteq S$ and $X$ was assumed not to be congruent with a subset of $E^{n}$. Therefore the $y_{i}$ 's form a metric basis, and the proof is complete.

Requiring that $M$ have the complete $n$-basis property allows Theorem 2 to be extended in the same way that Corollary 1.2 extends Theorem 1.

COROLLARY 2.1. $E^{n}$ is characterized among all complete and convex metric spaces having $n+1$ points not isometric with $n+1$ points of $E^{n-1}$ and the euclidean four point property by having the complete $n$-basis property. 
Proof. Let $M$ be a metric space with the hypotheses of the Corollary. By Theorem 2, $M$ is isometric with a subset of $E^{n}$ so we may think of $M$ as a subset of $E^{n}$. Assume $p$ is a point in $E^{n}-M$. By hypothesis, $M$ contains an $n$-simplex $S$ and the convex hull of $S$. Let $q$ be an interior point of the simplex and choose $x$ and $y$ on the line between $p$ and $q$ so that: (1) $q$ is the midpoint of $x$ and $y$, and (2) the two $n-1$ spheres, the endpoints of diameters of which are $x$ and $q$ and $q$ and $y$, are contained in $S$. Assume that $x$ is between $p$ and $q$. Let $f$ be an isometry between the two spheres such that $f(y)=q$ and $f(q)=$ $x$. Since each of the spheres contains an $n$-simplex, $f$ is an isometry which maps a complete metric basis of $M$ onto some subset of $M$. By the definition of a complete metric basis, $f$ may be extended to all of M. Let $g$ be that extension and note that $g(x)=f(x)$ where $f$ is defined (they are isometries in $E^{n}$ which coincide on an $n$-simplex). The composition mappings $g^{r}=g \circ g \circ \cdots \circ g \circ g$ are isometries of $M$ into $M$. By the Archimedean property of the reals there exists an $m$ such that $p$ is between $g^{m}(q)$ and $q$. The convexity of $M$ implies $p$ is in $M$, contradicting our choice of $p$. Therefore, $M=E^{n}$, completing the proof in this case. That $E^{n}$ has the properties of the Corollary is well known.

The euclidean four point property was shown by Wilson to characterize (separable) inner product spaces among the class of all complete, convex, externally convex metric spaces [7]. Our use of that property is based on the fact that it implies that $n$-point property for every natural number $n$ in a complete and convex metric space. Since Wilson, many authors have shown that weaker conditions than the euclidean four point property may be used in the characterization of inner product space (see [4], [6]). At least one of these, the weak euclidean four point property (the efpp for quadruples containing a linear triple), is strong enough to imply the $n$-point property for complete and convex metric spaces (see [1, p. 128]).

It can be shown that both the feeble and the queasy euclidean four point properties defined in [2], [3] imply the weak euclidean four point property in a complete and convex metric space. The conclusion of Theorem 2 will still be valid if the euclidean four point property is replaced by the weak, feeble or queasy form of that property. Corollary 2.1 may be extended to:

COROLlARY 2.2. $E^{n}$ is characterized among all complete and convex metric spaces having $n+1$ points not isometric with $n+1$ points of $E^{n-1}$ and the weak (feeble, queasy) euclidean four point property by having the complete $n$-basis property.

It would be interesting to know whether Corollary 2.2 is valid if weak, is replaced by any of the other properties used to characterize 
generalized euclidean space, i.e. isosceles, isosceles weak, isosceles feeble, external isosceles weak, etc. (a list may be found in [6]).

The complete $n$-point basis property is defined for all $n \geqq 0$ so, using the usual conventions about $E^{0}$ and $E^{-1}$ we have the following from Corollary 1.2:

COROLlary 2.3. For $n=0,1, E^{n}$ is characterized among all complete and convex metric spaces by the complete $n$-basis property.

Corollary 2.3 is not true for $n=2$. This may be seen from the following example. Let $M=X \cup Y$ where $X$ is the $x$-axis and $Y$ the $y$-axis in the usual cartesian plane. Define distance in $M$ to be the usual distance for two points on the same line and $x y=x \theta+\theta y$ where $\theta=(0,0)$. This space is complete, convex, has three points not isometric with three points of $E^{1}$ and every noncollinear triple is a complete metric bases, but it is not isometric with $E^{2}$.

\section{REFERENCES}

1. L. M. Blumenthal, Theory and applications of distance geometry, Oxford, 1953.

2. _ An extension of a theorem of Jordan and von Neumann, Pacific J. Math., 5 (1955), 161-167.

3. M. M. Day, On criteria of Kasahara and Blumenthal for inner product spaces, Proc. Amer. Math. Soc., 10 (1959), 92-100.

4. R. W. Freese, Criteria for inner product spaces, Proc. Amer. Math. Soc., 19 (1968), 953-958.

5. K. Menger, Untersuchungen über allgemeine Metrik, Math. Ann., 100 (1928), 75-163.

6. J. E. Valentine and S. G. Wayment, The euclidean symmetric isosceles queasy four point properties, Proc. Amer. Math. Soc., 34 (1972), 233-238.

7. W. A. Wilson, A relation between metric and euclidean spaces, Amer. J. Math., 54 (1932), 505-517.

8. - On the embedding of metric sets in euclidean space, Amer. J. Math., 57 (1935), 322-326.

Received July 2, 1974.

University of MAINE AT Orono 



\section{PACIFIC JOURNAL OF MATHEMATICS}

\section{EDITORS}

RICHARD ARENS (Managing Editor)

University of California

Los Angeles, California 90024

\author{
R. A. Beaumont \\ University of Washington \\ Seattle, Washington 98105
}

\section{J. DugundII}

Department of Mathematics

University of Southern California

Los Angeles, California 90007

D. Gilbarg and J. Milgram

Stanford University

Stanford, California 94305

\section{ASSOCIATE EDITORS}
E. F. BECKENBACH
B. H. NeumanN
F. WoLF
K. YoshidA

\section{SUPPORTING INSTITUTIONS}

\author{
UNIVERSITY OF BRITISH COLUMBIA \\ CALIFORNIA INSTITUTE OF TECHNOLOGY \\ UNIVERSITY OF CALIFORNIA \\ MONTANA STATE UNIVERSITY \\ UNIVERSITY OF NEVADA \\ NEW MEXICO STATE UNIVERSITY \\ OREGON STATE UNIVERSITY \\ UNIVERSITY OF OREGON \\ OSAKA UNIVERSITY
}

\author{
UNIVERSITY OF SOUTHERN CALIFORNIA \\ STANFORD UNIVERSITY \\ UNIVERSITY OF TOKYO \\ UNIVERSITY OF UTAH \\ WASHINGTON STATE UNIVERSITY \\ UNIVERSITY OF WASHINGTON \\ AMERICAN MATHEMATICAL SOCIETY
}

The Supporting Institutions listed above contribute to the cost of publication of this Journal, but they are not owners or publishers and have no responsibility for its contents or policies.

Mathematical papers intended for publication in the Pacific Journal of Mathematics should be in typed form or offset-reproduced (not dittoed), double spaced with large margins. Underline Greek letters in red, German in green, and script in blue. The first paragraph or two must be capable of being used separately as a synopsis of the entire paper. Items of the bibliography should not be cited there unless absolutely necessary, in which case they must be identified by author and Journal, rather than by item number. Manuscripts, in duplicate, may be sent to any one of the four editors. Please classify according to the scheme of Math. Reviews, Index to Vol. 39. All other communications should be addressed to the managing editor, or Elaine Barth, University of California, Los Angeles, California, 90024.

100 reprints are provided free for each article, only if page charges have been substantially paid. Additional copies may be obtained at cost in multiples of 50.

The Pacific Journal of Mathematics is issued monthly as of January 1966. Regular subscription rate: $\$ 72.00$ a year (6 Vols., 12 issues). Special rate: $\$ 36.00$ a year to individual members of supporting institutions.

Subscriptions, orders for back numbers, and changes of address should be sent to Pacific Journal of Mathematics, 103 Highland Boulevard, Berkeley, California, 94708.

PUBLISHED BY PACIFIC JOURNAL OF MATHEMATICS, A NON-PROFIT CORPORATION Printed at Jerusalem Academic Press, POB 2390, Jerusalem, Israel.

\section{Copyright (C) 1975 Pacific Journal of Mathematics All Rights Reserved}




\section{Pacific Journal of Mathematics

Vol. 60, No. $2 \quad$ October, 1975

Waleed A. Al-Salam and A. Verma, A fractional Leibniz q-formula ........... 1

Robert A. Bekes, Algebraically irreducible representations of $L_{1}(G) \ldots \ldots \ldots \ldots 11$

Thomas Theodore Bowman, Construction functors for topological

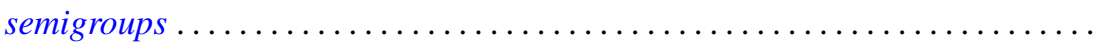

Stephen LaVern Campbell, Operator-valued inner functions analytic on the

closed disc. II .........................................

Leonard Eliezer Dor and Edward Wilfred Odell, Jr., Monotone bases in $L_{p} \ldots \ldots$.

Yukiyoshi Ebihara, Mitsuhiro Nakao and Tokumori Nanbu, On the existence of

global classical solution of initial-boundary value problem for

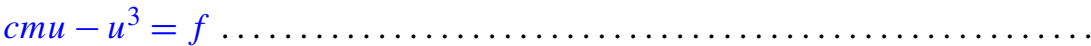

Y. Gordon, Unconditional Schauder decompositions of normed ideals of

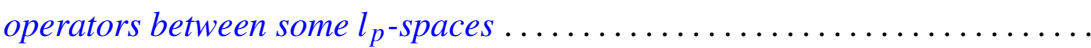

Gary Grefsrud, Oscillatory properties of solutions of certain nth order functional

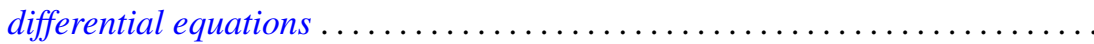

Irvin Roy Hentzel, Generalized right alternative rings ...................

Zensiro Goseki and Thomas Benny Rushing, Embeddings of shape classes of compacta in the trivial range .................................

Emil Grosswald, Brownian motion and sets of multiplicity . .

Donald LaTorre, A construction of the idempotent-separating congruences on a

bisimple orthodox semigroup .

Pjek-Hwee Lee, On subrings of rings with involution ...

Marvin David Marcus and H. Minc, On two theorems of Frobenius ...

Michael Douglas Miller, On the lattice of normal subgroups of a direct

product. .

Grattan Patrick Murphy, A metric basis characterization of Euclidean space

Roy Martin Rakestraw, A representation theorem for real convex functions ....

Louis Jackson Ratliff, Jr., On Rees localities and $H_{i}$-local rings ...

Simeon Reich, Fixed point iterations of nonexpansive mapping . .

Domenico Rosa, $B$-complete and $B_{r}$-complete topological algebras ...

Walter Roth, Uniform approximation by elements of a cone of real-valued

functions ....

Helmut R. Salzmann, Homogene kompakte projektive Ebenen

Jerrold Norman Siegel, On a space between $B H$ and $B_{\infty} \ldots$

235

Robert C. Sine, On local uniform mean convergence for Markov operators

James D. Stafney, Set approximation by lemniscates and the spectrum of an

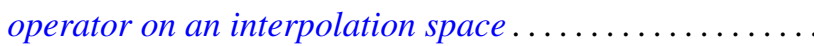

Árpád Száz, Convolution multipliers and distributions .......

Kalathoor Varadarajan, Span and stably trivial bundles ..........

Robert Breckenridge Warfield, Jr., Countably generated modules over

commutative Artinian rings....................... 